\title{
Block and Gradient Copoly(2-oxazoline) Micelles: Strikingly Different on the Inside
}

Sergey K. Filippov, ${ }^{* \dagger ®}$ Bart Verbraeken, ${ }^{\ddagger}$ Petr V. Konarev, ${ }^{\S} \|, \perp$ Dmitri I. Svergun, ${ }^{\S}$ Borislav Angelov, ${ }^{\#}$ Natalya S. Vishnevetskaya, ${ }^{\nabla}$ Christine M. Papadakis, ${ }^{\nabla} \odot$ Sarah Rogers, ${ }^{\bigcirc}$ Aurel Radulescu, ${ }^{\diamond}$ Tim Courtin, ${ }^{\dagger}$ José C. Martins, ${ }^{\dagger}$ Larisa Starovoytova, ${ }^{\dagger}$ Martin Hruby, ${ }^{\dagger}{ }^{\circ}$ Petr Stepanek, ${ }^{\dagger}$ Vitaly S. Kravchenko, ${ }^{\text {I }}$ Igor I. Potemkin, ${ }^{\mathbb{L}, \dagger \dagger}$ and Richard Hoogenboom* $*$

${ }^{\dagger}$ Institute of Macromolecular Chemistry, Academy of Sciences of the Czech Republic CZ - 16206 Praha 1, Czech Republic

${ }^{\ddagger}$ Department of Organic and Macromolecular Chemistry, Ghent University, Krijgslaan 281-S4, 9000 Ghent, Belgium

${ }^{\S}$ Hamburg Outstation, European Molecular Biology Laboratory c/o DESY, Notkestrasse 85, Hamburg 22607, Germany

"A.V. Shubnikov Institute of Crystallography, Federal Scientific Research Centre 'Crystallography and Photonics', Russian Academy of Sciences, Leninsky prospekt 59, Moscow 119333, Russian Federation

${ }^{\perp}$ National Research Centre "Kurchatov Institute", Akademika Kurchatova Place 1, Moscow 123182, Russian Federation

\#Institute of Physics, Academy of Sciences of the Czech Republic, 18221 Praha 8, Czech Republic

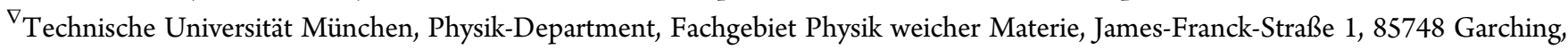
Germany

OISIS Facility, STFC, Rutherford Appleton Laboratory, Harwell Oxford, Didcot, OX11 0QX, United Kingdom

Forschungszentrum Jülich GmbH, Jülich Centre for Neutron Science JCNS, Outstation at Heinz Maier-Leibnitz Zentrum, Lichtenbergstraße 1, 85748 Garching, Germany

IThysics Department, Lomonosov Moscow State University, Moscow 119991, Russian Federation

${ }^{\dagger}$ National Research South Ural State University, Chelyabinsk 454080, Russian Federation

Supporting Information

ABSTRACT: Herein, we provide a direct proof for differences in the micellar structure of amphiphilic diblock and gradient copolymers, thereby unambiguously demonstrating the influence of monomer distribution along the polymer chains on the micellization behavior. The internal structure of amphiphilic block and gradient co poly(2-oxazolines) based on the hydrophilic poly(2-methyl-2-oxazoline) $(\mathrm{PMeOx})$ and the hydrophobic poly(2-phenyl-2-oxazoline) (PPhOx) was studied in water and water-ethanol mixtures by small-angle X-ray scattering (SAXS), smallangle neutron scattering (SANS), static and dynamic light scattering (SLS/DLS), and ${ }^{1} \mathrm{H}$ NMR spectroscopy. Contrast matching SANS experiments revealed that block copolymers form micelles with a uniform density profile of the core. In contrast to popular assumption, the outer part of the core of the gradient copolymer micelles has a distinctly higher density than the middle of the core. We attribute the latter finding to back-folding of chains resulting from hydrophilic-hydrophobic

interactions, leading to a new type of micelles that we refer to as micelles with a "bitterball-core" structure.
W ith the advent of living and controlled polymerization methods, the preparation of more and more complex polymer structures and architectures has become possible. ${ }^{1}$ Nowadays, star, graft, and even cyclic copolymers are very wellknown and well-investigated (co)polymer architectures. $^{2-4}$ Variation of monomer structures is mostly limited to block copolymers and statistical copolymers with close to random monomer distribution or minor composition drift along the chain. However, gradient copolymers that have a more gradual change in monomer composition are still largely unexplored. $^{5-15}$ In the present paper, we wish to shed light on the internal structure of nanoparticles composed of gradient

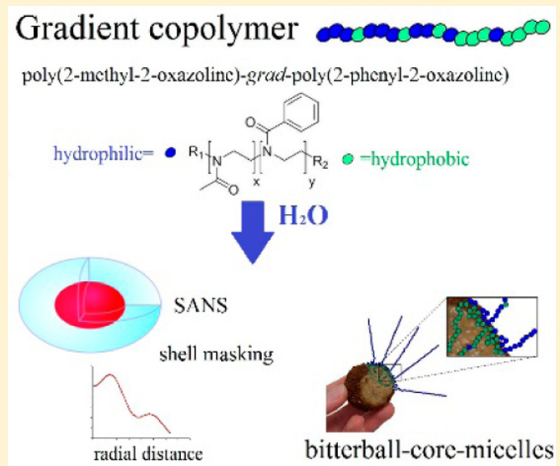


sphere model adequately fits to the experimental data, but only up to $q=0.4 \mathrm{~nm}^{-1}$ ( $q$ is the momentum transfer); there is no satisfactory fitting model describing the scattering at higher $q$ values (ref 18) that correspond in real space to the sizes of a few nanometers and smaller $(q \sim \pi / r)$. Thus, the description of gradient micelle structure on subnanometer scale is still missing.

The primary goal of the present work is, therefore, to investigate the internal structure of poly(2-methyl-2-oxazoline) (PMeOx) and hydrophobic poly(2-phenyl-2-oxazoline) $(\mathrm{PPhOx})$ gradient and block copolymer micelles (Scheme 1) with an unprecedented level of detail by SANS, including contrast variation using partially deuterated copolymers, and small-angle X-ray scattering (SAXS).

Scheme 1. Chemical Structure and Schematic Presentation of the Structures of the PMeOx-PPhOx Block and Gradient Copolymers Investigated in This Work
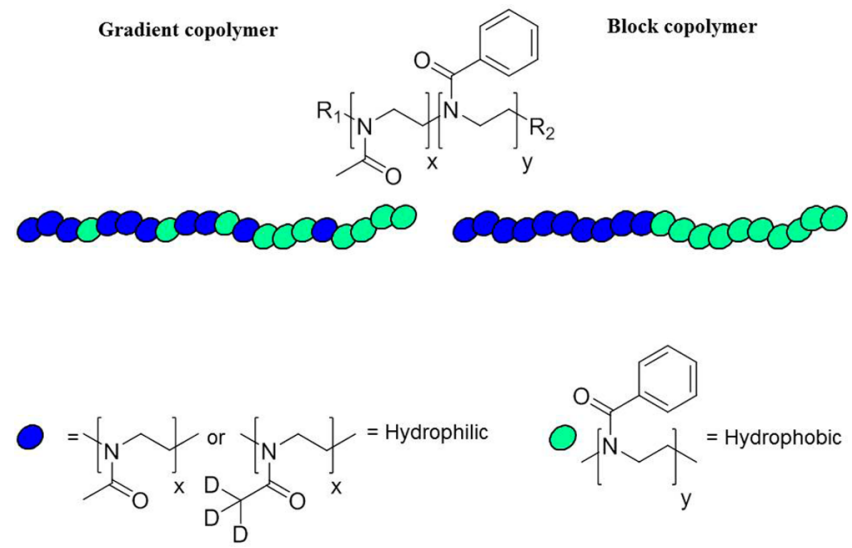

Block and gradient copolymers were synthesized according to previously reported methods (full experimental details are included in the Supporting Information). ${ }^{19,20}$ The chemical structure and composition of the synthesized copolymers are shown in Scheme 1 and are summarized in Table 1.

The self-assembly of the block and gradient copolymers was performed by direct dissolution in the solvents, and all studied block and gradient copolymers form nanoparticles in water and in water-ethanol mixtures, as manifested by the average $R_{\mathrm{h}}$

Table 1. Molecular Characteristics of the Copolymers

$\begin{array}{ccclcc}\text { sample }^{a} & \begin{array}{c}\mathrm{N}_{1} \\ (\mathrm{MeOx})\end{array} & \begin{array}{c}\mathrm{N}_{2} \\ (\mathrm{PhOx})\end{array} & \begin{array}{c}\text { solubility in } \\ \text { water }\end{array} & \begin{array}{c}M_{\mathrm{n}} \\ \mathrm{g} / \mathrm{mol}\end{array} & M_{\mathrm{w}} / M_{\mathrm{n}} \\ \text { 0-BH } & 100 & 0 & \text { soluble } & 11400 & 1.33 \\ \text { 0-BD } & 100 & 0 & \text { soluble } & 13200 & 1.35 \\ \text { I-BH } & 75 & 25 & \text { soluble } & 15900 & 1.09 \\ \text { I-GH } & 75 & 25 & \text { soluble } & 13500 & 1.17 \\ \text { I-BD } & 75 & 25 & \text { soluble } & 14300 & 1.30 \\ \text { I-GD } & 75 & 25 & \text { soluble } & 12000 & 1.22 \\ \text { II-BH } & 50 & 50 & \text { non soluble } & 14000 & 1.17 \\ \text { II-GH } & 50 & 50 & \text { non soluble } & 15300 & 1.18 \\ \text { II-BD } & 50 & 50 & \text { non soluble } & 15700 & 1.09 \\ \text { II-GD } & 50 & 50 & \text { non soluble } & 13200 & 1.12\end{array}$

${ }^{a} \mathrm{~B}$ stands for block and $\mathrm{G}$ for gradient copolymer, whereas $\mathrm{H}$ stands for protonated $\mathrm{MeOx}$ and $\mathrm{D}$ for a fully deuterated methyl group of $\mathrm{MeOx} ; \mathrm{N}_{1}$ and $\mathrm{N}_{2}$ are mole fractions of $\mathrm{MeOx}$ and PheOx, respectively. values which range from 10 to $50 \mathrm{~nm}$ (Supporting Information, Figure S1, S2, Table S1). In general, the nanoparticles formed by gradient copolymers are smaller than nanoparticles from block copolymers in the same solvent and at the same concentration and have higher critical micelle concentration (cmc) values (Supporting Information, Figures S3, S4).

To gain detailed insights into the micellar structures, SAXS and SANS experiments were conducted. The combined usage of SAXS and SANS is strongly advantageous for the study of the internal micellar structure since $\mathrm{X}$-rays and neutrons probe different scattering length densities (SLDs) (Supporting Information, Table S2, S3, Figure S5). The SAXS scattering data for both the block and gradient copolymers could be successfully fitted by a model of ellipsoidal block copolymer micelles (Figure 1, model B) as well as with a model of micelles with a rod-like homogeneous core with radially decaying density in the shell (Figure 1D, Figure S6, S7, Table S4). ${ }^{1,22}$

The fitting of SAXS data is further supported by SANS measurements of the nanoparticles in solvents where both $\mathrm{P}\left(d_{3}-\mathrm{MeOx}\right)$ and $\mathrm{PPhOx}$ have contrast. Figure 1 , left shows the $q$ dependence of the SANS intensity of neutron scattering for the copolymers I-GD and I-BD in $\mathrm{D}_{2} \mathrm{O}$ (solvent 1 , Table $\mathrm{S} 3$ ) and II-GD and II-BD in $40 \% d_{6}-\mathrm{EtOH}+60 \% \mathrm{D}_{2} \mathrm{O}$ (solvent 10 , Table S3).

In agreement with SAXS results, fitting of the scattering data by the models of core-shell ellipsoid (Figure 1, Model A) and spherical block copolymer micelle (Figure 1, Model C) gives a worse fit with the experimental data even when polydispersity is included in comparison with the rod-like model.

The size of the entire micelles and the micellar core as well as the aggregation number $N_{\mathrm{agg}}$, eccentricity, radial density exponent, and the solvent amount in the core were obtained by fitting of different form-factors mentioned above in various mixed solvents (Supporting Information, Figure S9, Table S5). The relatively large corona and radial density exponent (Figure S9f) is the most likely reflection of the long PMeOx chains in these copolymers. The micelles resulting from the block copolymers were found to be larger and have larger cores than the micelles from the gradient copolymers. SANS results demonstrated that the gradient copolymer micelles are smaller due to a lower aggregation number.

By using SANS and selective deuteration, scattering from the $\mathrm{PMeOx}$ was masked to reveal the presence of a micellar core. The $R_{\mathrm{g}}$ values found for these masked samples were lower than for solvents with unmasked PMeOx (Supporting Information, Figure S10a,b). In water, I-GD and I-BD form micelles with a core radius of 2.4 and $5.3 \mathrm{~nm}$ (Figure S11a). From the fitting of SANS data by a the form-factor of polydisperse ellipsoids, we can conclude that the micellar cores consisting of $\mathrm{PPhOx}$ have an anisotropic shape. The structural change for the core of the micelles consisting of II-BD and II-GD with increasing ethanol content is more evident from the corresponding pair-distance distribution function (PDDF) curves shown in Figure S11c,d, Supporting Information. The PDDF patterns from solutions with 20 to $50 \mathrm{vol} \%$ of ethanol exhibit a skewed bellshape, a typical feature of ellipsoidal particles. ${ }^{23}$ Increasing the ethanol content decreases the length of the core of ellipsoidal nanoparticles whereas asymmetry of the whole core increases.

The most striking difference between the inner structure of gradient and block micelles could be seen from the radial density profiles (Figure 2). This distribution is obtained from deconvolution of the PDDF functions (Supporting Information, Figure S9) assuming an ellipsoidal shape of the micelles. ${ }^{23}$ 

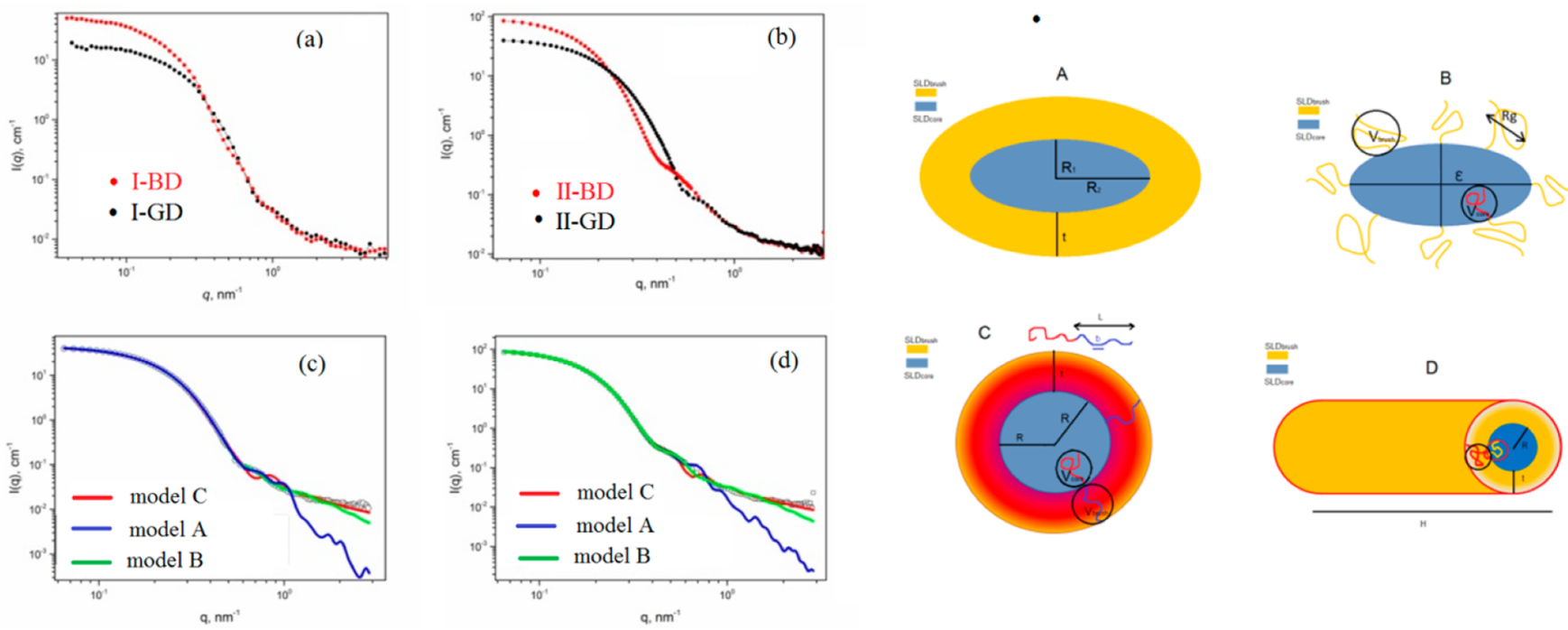

Figure 1. SANS data for (a) I-GD and I-BD in $\mathrm{D}_{2} \mathrm{O}$ (solvent 2, Table S3); (b) II-GD and II-BD in $\mathrm{D}_{2} \mathrm{O}$ (solvent 2, Table S3). (c) II-GD in $40 \% d_{6}$ EtOH $+60 \% \mathrm{D}_{2} \mathrm{O}$ (solvent 10) with different fits; (d) II-BD in $40 \% d_{6}$-EtOH+60\% $\mathrm{D}_{2} \mathrm{O}$ (solvent 10) with different fits. Scattering curves were extrapolated to infinite dilution. The models for SAXS and SANS data fitting; (A) core-shell ellipsoid, (B) elliptical block copolymer micelle with random walk chains, (C) spherical micelle with homogeneous core and radial decaying density shell with self-avoiding chains, and (D) rod-like micelle with rod-like homogeneous core and radial decaying density shell.
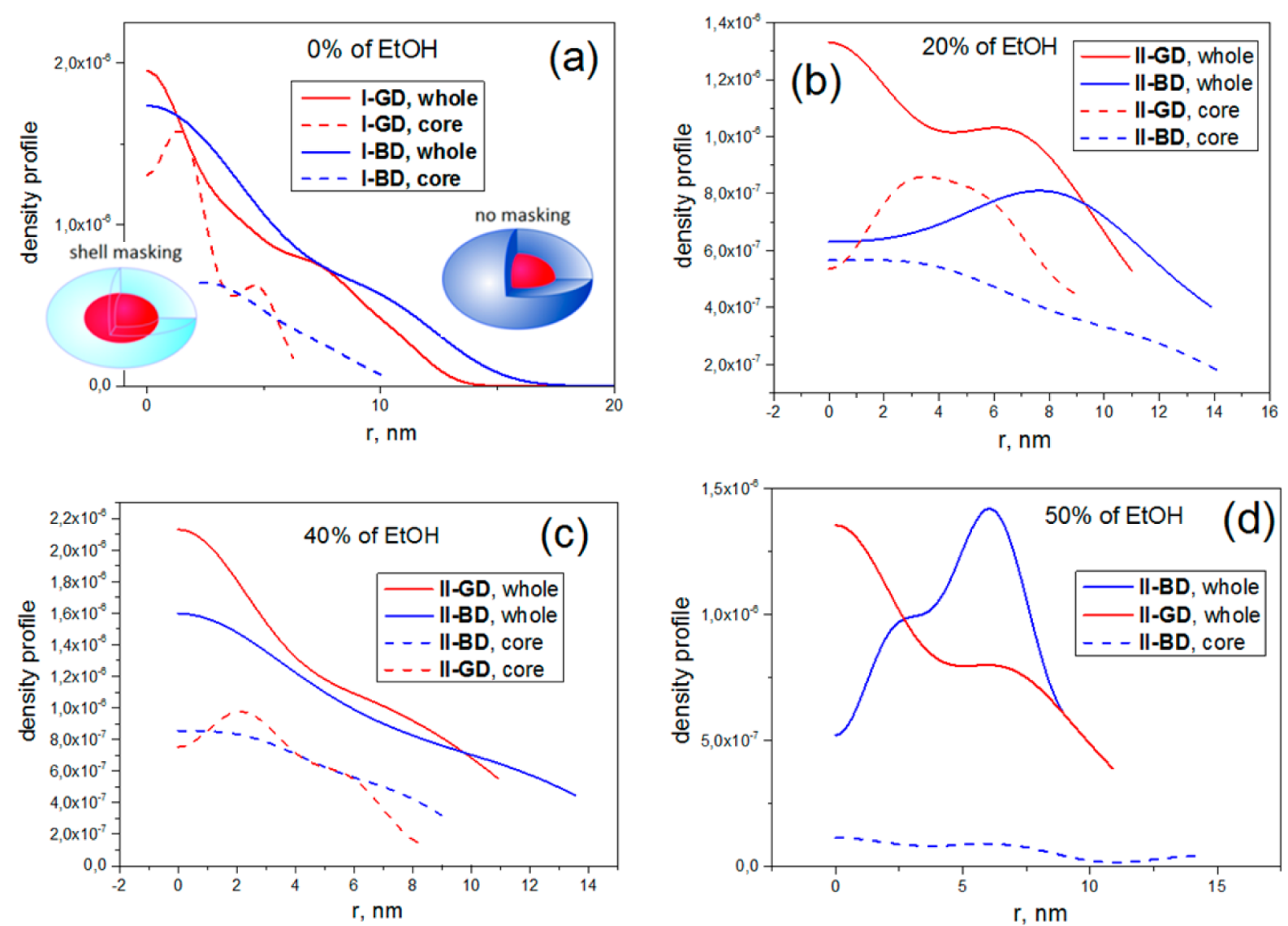

Figure 2. Contrast density profiles obtained by deconvolution of the PDDF shown in Figure S11c,d. (a) 0\% of EtOH; (b) $20 \%$ of EtOH; (c) $40 \%$ of $\mathrm{EtOH}$; (d) $50 \%$ of EtOH.

First the micelles of I-BD and I-GD copolymers in water will be discussed. When the scattering of $\mathrm{PMeOx}$ is not masked, the profile of radial density for both systems shows a typical coreshell structure with two-step behavior (Figure 2a). The dimension of the hydrophobic core can be assessed from the position of the plateau, yielding a value of $9 \mathrm{~nm}$ for micelles of I-BD and $6 \mathrm{~nm}$ for micelles of I-GD. These values are consistent with density profiles when $\mathrm{P}\left(d_{3}-\mathrm{MeOx}\right)$ is masked. Micelles of I-BD show almost constant behavior at low $r$ followed by a smooth decay. By contrast, nonmonotonous 
behavior with some oscillations is visible for micelles of the IGD copolymer. This effect is a direct proof of the nonhomogeneous core of the gradient copolymer micelles due to the presence of $\mathrm{PMeOx}$ repeating units inside the core and/or the formation of $\mathrm{PMeOx}$ loops from the $\mathrm{PPhOx}$ core. Importantly, such a structure of gradient copolymer micelles with loops of the hydrophilic parts was very recently predicted in a theoretical paper, albeit never experimentally observed. ${ }^{24}$ It is reasonable to assume that some $\mathrm{PMeOx}$ units will be present in the PPhOx core since they are jammed in-between $\mathrm{PPhOx}$ repeat units. The radial density profiles for micelles consisting of II-BD and II-GD in water with $40 \mathrm{vol} \%$ of ethanol revealed a similar structure for the gradient and block micelles (Figure 2c). Again, micelles composed of the block copolymer manifest the typical core-shell features, whereas gradient copolymer micelles possess a completely different behavior with some density oscillation inside the core. This gradient copolymer micellar core-structure with higher density outside and more liquid-like structure inside resembles the structure of a "bitterball" (Figure 3). Therefore, we suggest a new term for

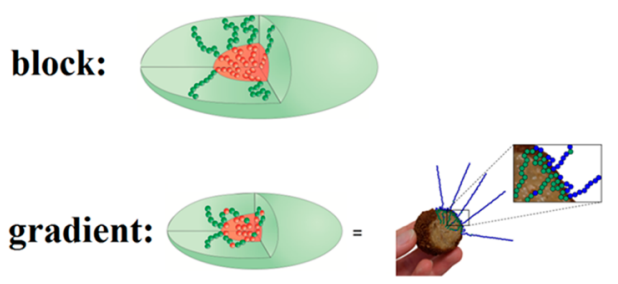

Figure 3. Hypothetical structure of block and gradient nanoparticles and bitterball-core-micelles. Red color stands for $\mathrm{PhOx}$ and green stands for $\mathrm{MeOx}$ blocks.

such types of gradient copolymer micelles that have denser outer layer of the core compared to the inside - "bitterballcore-micelles". For $20 \%$ ethanol, the core of the gradient copolymer micelles also indicates inhomogeneity and formation of "bitterball-core-micelles" (Figure 2). Surprisingly, we observed for the unmasked II-BG copolymer micelles that the radial profile also increases with increasing distance $r$; meanwhile, the core of masked block copolymer shows classic behavior. We are inclined to explain this by preferential absorption of ethanol inside the micellar core. The presence of a larger amount of ethanol results in swelling and distortion of the interior of the micelles.

The differences in the core density of the block and gradient copolymer micelles suggest stronger phase segregation in the block copolymer micelles leading to enhanced dehydration of the core. This conclusion is additionally supported by ${ }^{1} \mathrm{H}$ NMR spectroscopic data where the spin-spin relaxation time $T_{2}$ was measured for I-GD and I-BD gradient and block copolymers in $\mathrm{D}_{2} \mathrm{O}$ and $d_{4}$-methanol. The obvious conclusion we can make is that $\mathrm{PPhOx}$ is completely shielded and hidden inside the core for block copolymer micelles in $\mathrm{D}_{2} \mathrm{O}$ since no PPhOx signal can be observed (Supporting Information, Figure S24, Table S6). For gradient copolymer micelles in $\mathrm{D}_{2} \mathrm{O}$, however, some $\mathrm{PhOx}$ groups are still mobile and can be observed, proposedly because they are present in a $\mathrm{PMeOx}$ rich part of the chain in the shell. In addition, the mobility of $\mathrm{PMeOx}$ in the micellar structures is retarded in comparison to $\mathrm{PMeOx}$ homopolymer in $\mathrm{D}_{2} \mathrm{O}$.

In conclusion, the monomer distribution (gradient vs block) is of primary importance on the structure of micelles formed by $\mathrm{PMeOx}-\mathrm{PPhOx}$. The micelles resulting from the block copolymers are bigger than the ones from the gradient copolymers based amphiphilic block and gradient copolymers. The micelles resulting from the gradient copolymers have different eccentricity, aggregation number, core sizes, solvent volume fraction, and radial density profile than the micelles resulting from the block copolymers. Contrast-matching SANS experiments prove that gradient copolymer micelles have a unmonotonous profile of the core, whereby the outer part of the core of the gradient copolymer micelles has a distinctly higher density than the core itself.

\section{ASSOCIATED CONTENT}

S Supporting Information

The Supporting Information is available free of charge on the ACS Publications website at DOI: 10.1021/acs.jpclett.7b01588.

Concentration dependence of $R_{\mathrm{h}}$, molecular weights, aggregation numbers and second virial coefficients measured by static light scattering, additional SAXS/ SANS data, concentration dependence of $R_{\mathrm{g}}$ of core and whole particles for different content of ethanol, normalized FCS correlation functions and results and ITC curves, fitting parameters from SANS experiments by different models, and NMR and GPC spectra used to determine copolymer structure (PDF)

\section{AUTHOR INFORMATION}

\section{Corresponding Authors}

*Tel: +420-608720561; Fax: +420-296809410; E-mail: sfill225@gmail.com (S.K.F.).

*E-mail: richard.hoogenboom@ugent.be (R.H.).

ORCID

Sergey K. Filippov: 0000-0002-4253-5076

Christine M. Papadakis: 0000-0002-7098-3458

Martin Hruby: 0000-0002-5075-261X

Igor I. Potemkin: 0000-0002-6687-7732

Richard Hoogenboom: 0000-0001-7398-2058

\section{Notes}

The authors declare no competing financial interest.

\section{ACKNOWLEDGMENTS}

EMBL (Hamburg), ILL (Grenoble), KWS-2 at JCNS (Garching), and STFC at ISIS (Didcot, UK) are acknowledged for beam time allocation. R.H. and B.V. acknowledge the Flemish agency for Innovation through Science and Technology (IWT). S.F. and R.H. acknowledge the support of the mobility project AV ČR-FWO, FWO-17-05. S.F. and B.A. acknowledge the support of the Czech Science Foundation GACR (Grant 17-00973S). M.H. acknowledges financial from the Czech Grant Foundation (Grant \#16-03156S) and from the Ministry of Health of the Czech Republic (Grant \#15-25781a). S.F. thanks Dr. Cernoch for measurements of solvent densities. V.S.K. acknowledges financial support from the Russian Foundation for Basic Research. I.I.P. thanks the Russian Science Foundation, Project No. 15-13-00124, for financial support. The work was supported by the Government of the Russian Federation within Act 211, Contract No. 02.A03.21.0011. We thank Deutscher Akademischer Austausch Dienst for travel support within the program Projektbezogener Personenaustausch-PPP. The $700 \mathrm{MHz}$ was funded by the FFEU-ZWAP initiative of the Flemish Government and a Hercules grant (AUGE09/006). 


\section{REFERENCES}

(1) Matyjaszewski, K.; Müller, A. H. E. 50 years of living polymerization. Prog. Polym. Sci. 2006, 31, 1039-1040.

(2) Pitsikalis, M.; Pispas, S.; Mays, J. W.; Hadjichristidis, N. Nonlinear Block Copolymer Architectures. Adv. Polym. Sci. 1998, 135, 1-137.

(3) Polymeropoulos, G.; Zapsas, G.; Ntetsikas, K.; Bilalis, P.; Gnanou, Y.; Hadjichristidis, N. 50th Anniversary Perspective: Polymers with Complex Architectures. Macromolecules 2017, 50, $1253-1290$.

(4) Hadjichristidis, N.; Pispas, S.; Floudas, G. Block Copolymers: Synthetic Strategies, Physical Properties, and Applications; John Wiley \& Sons: Hoboken, NJ, 2003.

(5) Davis, K. A.; Matyjaszewski, K. Statistical, Gradient, Block and Graft Copolymers by Controlled/Living Radical Polymerizations; Springer: Berlin, 2012.

(6) Mok, M. M.; Kim, J.; Torkelson, J. M. Gradient Copolymers with Broad Glass Transition Temperature Regions: Design of Purely Interphase Compositions for Damping Applications. J. Polym. Sci., Part B: Polym. Phys. 2008, 46, 48-58.

(7) Steinhauer, W.; Hoogenboom, R.; Keul, H.; Moeller, M. Block and Gradient Copolymers of 2-Hydroxyethyl Acrylate and 2Methoxyethyl Acrylate via RAFT: Polymerization Kinetics, Thermoresponsive Properties, and Micellization. Macromolecules 2013, 46, $1447-1460$.

(8) Borisova, O.; Billon, L.; Zaremski, M.; Grassl, B.; Bakaeva, Z.; Lapp, A.; Stepanek, P.; Borisov, O. pH-Triggered Reversible Sol-Gel Transition in Aqueous Solutions of Amphiphilic Gradient Copolymers. Soft Matter 2011, 7, 10824-10833.

(9) Borisova, O.; Billon, L.; Zaremski, M.; Grassl, B.; Bakaeva, Z.; Lapp, A.; Stepanek, P.; Borisov, O. Synthesis and pH- and SalinityControlled Self-assembly of Novel Amphiphilic Block-Gradient Copolymers of Styrene and Acrylic acid. Soft Matter 2012, 8, 76497659.

(10) Černochová, Z.; Bogomolova, A.; Borisova, O. V.; Filippov, S. K.; Černoch, P.; Billon, L.; Borisov, O. V.; Štěpánek, P. Thermodynamics of the Multi-Stage Self-Assembly of $\mathrm{pH}$-Sensitive Gradient Copolymers in Aqueous Solutions. Soft Matter 2016, 12, 6788-6798.

(11) Zheng, C.; Huang, H.; He, T. Micellization of St/MMA Gradient Copolymers: a General Picture of Structural Transitions in Gradient Copolymer Micelles. Macromol. Rapid Commun. 2013, 34, $1654-1661$.

(12) Zheng, C.; Huang, H.; He, T. Gradient Structure-Induced Temperature Responsiveness in Styrene/Methyl Methacrylate Gradient Copolymers Micelles. Macromol. Rapid Commun. 2014, 35, 309316.

(13) Van Steenberge, P. H. M.; Verbraeken, B.; Reyniers, M.-F.; Hoogenboom, R.; D'hooge, D. R. Model-Based Visualization and Understanding of Monomer Sequence Formation in Gradient Copoly(2-oxazoline)s on the Basis of 2-Methyl-2-Oxazoline and 2Phenyl-2-Oxazoline. Macromolecules 2015, 48, 7765-7773.

(14) Milonaki, Y.; Kaditi, E.; Pispas, S.; Demetzos, C. Amphiphilic Gradient Copolymers of 2-Methyl- and 2-Phenyl-2-Oxazoline: Selforganization in Aqueous Media and Drug Encapsulation. J. Polym. Sci, Part A: Polym. Chem. 2012, 50, 1226-1237.

(15) Pippa, N.; Kaditi, E.; Pispas, S.; Demetzos, C. Gradient Block Copolymer Structures as Drug Nanocarriers. Adv. Sci., Eng. Med. 2014, $6,642-648$.

(16) Okabe, S.; Seno, K.; Kanaoka, S.; Aoshima, S.; Shibayama, M. Small-angle Neutron Scattering Study on Block and Gradient Copolymer Aqueous Solutions. Polymer 2006, 47, 7572-7579.

(17) Okabe, S.; Fuse, C.; Sugihara, S.; Aoshima, S.; Shibayama, M. Structural Transition in Block and Gradient Copolymer Aqueous Solutions. Phys. B 2006, 385-386, 756-758.

(18) Okabe, S.; Seno, K.; Kanaoka, S.; Aoshima, S.; Shibayama, M. Micellization Study on Block and Gradient Copolymer Aqueous Solutions by DLS and SANS. Macromolecules 2006, 39, 1592-1597.
(19) Hoogenboom, R.; Thijs, H. M. L.; Wouters, D.; Hoeppener, S.; Schubert, U. S. Solvent Responsive Micelles Based on Block and Gradient Copoly(2-oxazoline)s. Macromolecules 2008, 41, 1581-1583.

(20) Kuhn, P.; Weberskirch, R; Nuyken, O.; Cevc, G. Synthesis of Segmentally Deuterated Poly(acetyliminoethylene) Lipopolymers, Material for Neutron Scattering and NMR Studies. Des. Monomers Polym. 1998, 1, 327-346.

(21) Breßler, I.; Kohlbrecher, J.; Thünemann, A. F. SASfit: a Tool for Small-Angle Scattering Data Analysis Using a Library of Analytical Expressions. J. Appl. Crystallogr. 2015, 48, 1587-1598.

(22) Pedersen, J. S.; Hamley, I. W.; Ryu, Ch. Y.; Lodge, T. Contrast Variation Small-Angle Neutron Scattering Study of the Structure of Block Copolymer Micelles in a Slightly Selective Solvent at Semidilute Concentrations. Macromolecules 2000, 33, 542-550.

(23) Glatter, O.; Kratky, O. Small-Angle X-ray Scattering; Academic Press: London, 1982.

(24) Kravchenko, V. S.; Potemkin, I. I. Micelles of Gradient vs Diblock Copolymers: Difference in the Internal Structure and Properties. J. Phys. Chem. B 2016, 120, 12211-12217. 